\title{
L’hybridité de la lettre galante, ou la transgression innovante
}

Saturée de sens jusqu'au point d'en être vidée, la notion d'hybridité a suscité depuis les années 1980 un vif intérêt chez des chercheurs de divers horizons. Dès le tournant du siècle, les nombreux ouvrages au sujet de l'hybridité essaient mieux cerner les contours de l'hybride dans des domaines spécifiques, aussi variés que les neurosciences $^{1}$ et les postcolonial studies ${ }^{2}$. Dans le champ littéraire, Reynier (2004 : 55-6) ainsi que Budor et Geerts (2004: 12) caractérisent le texte hybride par la transgression des limites entre deux ou plusieurs voix et formes, entre plusieurs styles, traditions et genres, littéraires ou non. Cette fusion originale engendre la création de nouveaux sens et de nouveaux objets littéraires. Par conséquent, l'hybridité littéraire s'est maintes fois avérée être une source inépuisable de curiosités et de surprises. Son importance va cependant bien au-delà des délectables épiphanies littéraires. L'hybridité dans les Belles-Lettres aide non seulement à tracer les évolutions dans le champ littéraire, mais elle stimule en même temps l'inventivité créatrice.

Pour considérer ces dimensions révélatrice et expérimentale de l'hybridation littéraire, nous aimerions retourner à une époque où l'hybridité littéraire en tant que concept n'existait point encore, mais était bel et bien présente. Bien que nombre de critiques associent l'hybridation à la postmodernité, ce seront ici les lettres galantes produites à l'Hôtel de Rambouillet (1608-1665) qui nous invitent à revoir et prendre pour outil la notion même d'hybridité. Dans ce premier salon mondain célèbre non seulement à Paris mais à travers toute la France, Catherine de Vivonne, Marquise de Rambouillet (1588-1665), accueillait une société mixte d’aristocrates, d’avocats

Astrid Van Assche - doctorante au département de littérature française de l'Université de Gand. Adresse pour correspondance : Université de Gand, Blandijnberg 2, 9000 Gand, Belgique ; e-mail : astrid. vanassche@ugent.be

1. Voir A. Schmitt, «Cognition et Hybridité. Le lecteur dans la zone grise », Cahiers de Narratologie, $\mathrm{n}^{\circ} 28,2015$, https://narratologie.revues.org/7201.

2. Voir R. Young, Colonial Desire: Hybridity in Theory, Culture and Race, London, Routledge, 1995. 
et d'écrivains majeurs tels que Malherbe et Corneille, mais encore Marie de RabutinChantal, célèbre marquise de Sévigné et Marie-Madeleine Pioche de La Vergne, Madame de La Fayette (Lathuillère, 1962 : 65). Dans l'Hôtel régnait une fascination pour la conversation raffinée et la lettre galante, inextricablement liée à la sociabilité du XVII siècle, y profitait d'une même valorisation.

Nous étudierons dans cet article l'hybridité au sein des lettres d'une première génération de galants épistoliers qui a vu le jour à l'Hôtel de Rambouillet. Dans notre corpus, qui consiste en les lettres galantes d'entre autres Vincent Voiture (1597-1648), Charles Cotin (1604-1681) et Antoine Godeau (1605-1672), l'hybridité est au centre de l'entreprise poétique. Nous regarderons d'abord en quoi la forme des lettres galantes peut être dite hybride, pour ensuite analyser l'hybridité des auteurs et de l'esthétique galante. L'étude de ces trois aspects nous permettra d'évaluer dans quelles mesures l'hybridité littéraire documente en effet les évolutions en œuvre dans le champ littéraire de l'époque et s'y manifeste en tant que moteur d'inventivité créatrice.

\section{L'hybridité de la forme épistolaire : la lettre galante en tant qu'espace indéfini}

\subsection{Entre manuscrit et discours performatif}

Contrairement à ce que nous nous attendons dans le cas d'une correspondance, la correspondance au XVII ${ }^{e}$ siècle avait avant tout une fonction mondaine éminemment sociale. À l'Hôtel de Rambouillet, l'échange des lettres était un passage obligé de la relation entre les épistoliers d'une part, et de l'autre, les aristocrates dont ils recherchaient l'approbation et le soutien. Les lettres galantes circulaient dans les cabinets et les salons, passées de main en main. Le partage des lettres était à l'époque tellement courant que les épistoliers devaient stipuler explicitement s'ils ne voulaient pas que leur lettre circule. Le Comte d'Avaux prie ainsi «très-sérieusement » son destinataire de veiller à ce que la lettre « ne sorte point de [ses] mains" (Roux, $1858: 27)^{3}$.

En tant que médium social, les lettres galantes étaient souvent lues à voix haute pour animer les réunions de salon. Cette lecture collective soulève d'emblée la problématique du rapport de la lettre à l'oralité et, nous y reviendrons, à l'altérité. La lettre (écrite) s'inspirait de la conversation mondaine (orale), dont elle imite la spontanéité (oralité écrite). Une correspondante anonyme demande par exemple à Charles Cotin de lui transcrire ses «beaux raisonnements » sur les Songes : «Vous en parlâtes si bien, que je serais ravie de les voir après les avoir entendus » (Cotin, $1663: 276$ ). Ces traces écrites de la conversation mondaine étaient ensuite polies en fonction de la lecture collective. C'est dans cette mouvance du message utilitaire au discours performatif que se développe le genre épistolaire, longtemps minoré en tant que genre littéraire à cause de son statut de moyen de communication avant tout privé.

3. Lorthographe des fragments de lettres et de poésie ici présentés a été modernisée. 
Comme ils imitent la spontanéité de la conversation mondaine, les galants épistoliers sopposent aux processus de modélisation rigoureux des doctes, au pédantisme et au style châtié. Il ne faut cependant pas se tromper sur la revendication de spontanéité des épistoliers mondains. Tout comme les galants épistoliers peuvent descendre jusqu'au langage familier et badin des plaisanteries, ils peuvent sélever à la cérémonie et l'emphase. Le lecteur attentif découvre alors la "délicatesse » et "l'ingéniosité » de ces lettres tout de même destinées à l'applaudissement (Denis, 1998 : 58). Il s'agit donc d'un mélange intriguant dans lequel les belles tournures de phrases et les constructions rhétoriques sont joliment camouflées en faveur d'une soi-disant spontanéité et d'une désinvolture toute travaillée.

\subsection{Entre simple instrument usuel et composition littéraire}

Les lettres des membres de l'Hôtel de Rambouillet informent, flattent, remercient, réprimandent et consolent. En ce faisant, elles font appel entre autres aux genres non-littéraires du petit mot, du journal intime et du récit de voyage. Au moment où les lettres mondaines gagnent alors en épaisseur littéraire suite à la présence de plus en plus marquée du « public » des lectures collectives, cette hybridation des registres utilisés se double d'une dynamique entre discours littéraires d'une part, et non-littéraires de l'autre. Cette dualité s'inscrit dans l'idée de paternité dans l'histoire littéraire qu'adopte Todorov : " chaque type de discours qualifié habituellement de littéraire a des "parents" non littéraires qui lui sont plus proches que tout autre type de discours "littéraire" »(1987: 10). Ainsi, la notion d’hybridité se présente à la critique littéraire comme un atout dans l'analyse et la description des genres en formation.

\section{L'hybridité identitaire : l'entre-deux, l'entre trois}

\subsection{L'auteur comme porte-parole et interprète}

Pour ce qui est de la figure du galant épistolier, elle est loin de l'hybridité identitaire typiquement postmoderne de la fragmentation du moi au sein d'une enquête psychologique. La réussite d'une lettre repose largement sur l'adaptation à la personnalité du destinataire. Comme le galant épistolier a tout intérêt à s'adresser à plusieurs personnes à la fois, il adopte une identité malléable, selon les goûts et les aspirations de la collectivité du cercle mondain. Porte-parole de cette belle société, la voix de lépistolier ne révèle donc guère l'intimité du moi ; elle est portée par l'entrecroisement des voix des habitués du salon, dont elle transcrit l'éthos. L'hybride ne menace donc pas ici l'unité identitaire du sujet, mais il est investi au contraire pour renforcer l'unité du groupe, en affirmant les connaissances culturelles partagées du groupe et en en transmettant des idéaux en mutation.

La figure du galant épistolier, scripteur hétéroclite, réunit ainsi le " moi » (potentiellement multiple, voire collectif), l'« autre » (le destinataire individuel auquel la lettre est adressée officiellement et les " autres » (l'auditoire/lectorat réel, assistant 
aux réunions mondaines). Toutes les facettes de cette triple figure sont donc porteuses d'une tension entre individualité et collectivitét. Cette multiplicité peut se concrétiser jusque dans l'endossement des identités de fantaisie, tels l'Africain pour Voiture, et l'Hermite Mitre pour Godeau. Dans une lettre à Cotin, ses co-membres de l'Hôtel sadressent au "pasteur Aristée » et signent " les Bergers et Bergères de la Foret de Marchenoir» (Cotin, 1663 : 82-83). Ces signatures hybrides permettent aux épistoliers d'expérimenter librement avec d'autres réalités (historiques et géographiques), ainsi que d'autres thèmes, styles et images.

\subsection{L'auteur sans autorité}

L'hybridité identitaire de lépistolier même se double d'un statut d'auteur fort ambigu. De même que la lettre nétait pas à lépoque considérée comme proprement littéraire, les galants épistoliers évitent de se dire auteur ou professionnel de lettres. Ils préfèrent revendiquer le statut d' "écrivain amateur ${ }^{5}$, ce qui s'inscrit parfaitement dans la logique nobiliaire régnant au début du XVII e siècle de l'otium literatum, ou loisir lettré. Lattitude désinvolte des galants épistoliers par rapport à la qualité d’auteur leur a valu le nom paradoxal d'" auteurs sans autorité » (Denis, $2001: 141$ ). Cette désinvolture nétait en fin de compte qu'une façade, voire une stratégie : leurs lettres leur servaient bel et bien de carte de visite, voire de source de récompenses financières et autres.

\section{L'hybridité de l'esthétique galante : du pêle-mêle à la pollinisation croisée}

\subsection{Hybridité générique : mélanges et métissages}

Nicolas Balutet s'appuie sur le riche héritage de Mikhail Bakhtine, Julia Kristeva, Roland Barthes et Gérard Genette pour définir l'hybridité générique, qui correspond à la " coexistence » de plusieurs genres littéraires, extralittéraires ou artistiques au sein d'un seul texte. Il ne s'agit pas d'un effacement, mais plutôt d'un éclatement des genres, comme on le constate par exemple dans le roman, qui «s'approprie toutes les formes d'expression » et " exploite à son profit tous les procédés " (Robert, 1972 : 14). Les réflexions critiques de Julika Griem (1998), Michael Sinding (1995) et Martina Allen (2013) sur l'hybridité, nous invitent à mettre en garde contre l'idée réductrice de la coexistence de genres comme un mélange (impur) de deux genres (purs) hétérogènes. Cette conception de genre fort essentialiste nie la diversité et l'adaptabilité des genres, qui ne sont point stables, ni naturellement distincts l'un de l'autre. Les sous-titres de cet article l'ont démontré : l'hybridité, comme la lettre galante, focalise sur la diversité et la multiplicité, bien au-delà des oppositions binaires.

4. La multiplicité du sujet épistolaire, ainsi que la portée potentielle d'une lettre, sont abordées dans toute leur richesse dans La carte postale, de Socrate à Freud et au-delà de Jacques Derrida.

5. Voir A. Viala, Naissance de l'écrivain. Sociologie de la littérature à l'âge classique, Paris, Minuit, 1985. 
Bien que la forme épistolaire soit souvent associée à la sincérité et l'objectivité, les lettres galantes présentent un discours pris entre deux régimes : celui de l'information objective sur la vie mondaine et celui d'une reprise fictionnelle. Les membres du salon cherchent leurs reflets dans le miroir enchanté que leur tendent ces fictions divertissantes. Dans sa correspondance, Voiture représente la Marquise de Rambouillet et ses filles comme des anges auréolés de lumière, "qui semblaient ne faire que descendre du Ciel » (1691: 8). Décrivant le souper de lélégante société Rambouillet, son regard se pose sur la table éclairée de mille bougies, qui « semble être servie par des fées » (Voiture, $1855: 47)$. Non sans imagination, Voiture raconte avoir vu dans le jardin de l'Hôtel « un tel nombre de feux d'artifice, qu'il semblait que toutes les branches et les troncs d'arbre se convertissent en fusées " (Voiture, $1855: 47$ ).

Ce mélange orchestré des faits réels et féériques au sein de la lettre galante nous rappelle ce que Serge Doubrovsky désignait en 1977 comme de l'autofiction. Lautofiction, forme hybride, déstabilise l'opposition vérité/fiction et fait appel à la fois au pacte référentiel de l'autobiographie et à la fictivité, associée au pacte romanesque. Voiture, co-auteur d'un roman qui restera inachevé, L'histoire d'Alcidalis et de Zélide, fait lui-même allusion à la concurrence entre l'autofiction épistolaire et l'autofiction romanesque quand il flatte Julie de Rambouillet en lui écrivant que "la moindre de [ses] Lettres vaut mieux que Zélide et Alcidalis avec leurs Royaumes » (Boyer, $1701: 119)$.

Les louanges des galants épistoliers sont visiblement nourries par la littérature chevaleresque et pastorale, dotées d'éléments issus des fééries, des fables et légendes, sans pour autant relever de l'une ou l'autre, ou de plusieurs, de ces catégories. En outre, les genres non littéraires tels le récit de voyage, le journal (intime) et le petit mot impriment leur marque sur la lettre galante. Noëlle Batt indique à juste titre que dans le texte hybride, toutes les "modalités hétérogènes » sont engagées dans la " coopération » (Batt, 2001 : 75-9). Dans la même veine, Budor et Geerts parlent de l'infini potentiel créateur de la réunion déléments « disparates mais compatibles » et de la proclamation du « composite » (2004: 13). Le galant épistolier se sert de tous les registres et de toutes les ressources pour mieux louer, remercier, divertir ou consoler dans sa correspondance. Écoutons Godeau qui se sert des images du genre merveilleux pour assurer Madame Desloges de ne la jamais oublier, quoiqu'il arrive :

Quand l'air y serait aussi pur qu'il était à la naissance du monde, quand de toutes les saisons on n'y connaitrait que le Printemps, que les rivages des fleuves seraient semés de perles, que les arbres auraient des feuilles d’or, enfin quand il posséderait plus de raretés que l'Isle d'Urgande ${ }^{6}$, ou d'Armide ${ }^{7}$, rien ne pourrait mồter le souvenir de ce que j’ai perdu (Faret, $1638: 529$ ).

6. Urgande, la bonne fée dans L'histoire d'Amadis de Gaule de Quinault.

7. Armide, la magicienne dans la tragédie Armide de Lully et Quinault. 
Cette première hybridité dans l'esthétique galante a engendré bien d'autres innovations littéraires, que nous découvrirons sous la forme de l'hybridité dialogique.

\subsection{Hybridité dialogique : croisements et contaminations}

L'hybridité dialogique vise « la transformation dans un texte particulier de différents éléments culturels, littéraires et linguistiques pris dans d'autres textes " (Balutet, 2016 : n.p.). Songeons aux allusions et aux imitations intertextuelles, aussi diverses que la citation et la parodie. Marc Eigeldinger parle justement d'un "travail d'appropriation et de réécriture qui s'applique à recréer le sens » (1987 : 11). L'hybridité dialogique surgit alors aux moments où les attentes du lecteur sont délibérément tronquées, ceci par une association peu conventionnelle ou ironique d'éléments génériques que Sinding a dénommée conceptual blending (1995 : 592). Jacques Derrida faisait déjà allusion à l'hybridité dialogique en désignant sa loi du genre comme ouverte à la transgression, plus précisément comme "un principe de contamination, une loi d'impureté, une économie du parasite » (1986:256).

Grâce à la littérarité non encore définie du genre épistolaire et la grande liberté de son scripteur, ladite transgression derridienne est facilement repérable dans les lettres galantes. Elle y apparaît sous les formes diverses de renversements des louanges traditionnelles, de métaphores insolites, de sous-entendus gaillards et de fictions ingénieuses, brouillant les conventions génériques au travers d'un jeu sur la trinité de l'ironique, l'implicite et l'intertextuel. Les galants épistoliers nous surprennent en rassemblant sur le support de la lettre la réalité et la fiction, la prose et la poésie, le sérieux et le frivole. Voiture renverse par exemple léloge traditionnel, quand il loue le Comte de Guiche qui aurait selon Voiture surpassé en combat les héros de livres. Il y ajoute à la fin qu' " à cette heure [...] l'invention des armes enchantées est perdue, et que la coutume n'est plus que les Héros soient invulnérables [...] » $(1691: 228)$. Non seulement Voiture renverse l'usage des louanges hyperboliques inspirées des romans héroïques, mais, en outre, il indique une tendance plus large dans la littérature de son époque vers l'approfondissement du personnage.

Les clichés issus des genres en vogue tels que la poésie pétrarquiste et le roman pastoral et héroïque sont souvent abordés dans les lettres galantes pour en tirer des effets surprenants et cocasses. Charles Cotin explore la polysémie des mots et des images appartenant au script de l'amour courtois en jouant par exemple sur le motif pétrarquiste de comparer l'aimée au soleil. Ainsi explique-t-il à Mademoiselle de Rohan, quelle séjourne trop dans ses propriétés en Guyenne et en Bretagne pour y régner. Il lui adresse le suivant message pour la convaincre de demeurer à Paris :

À cause qu'on vous a dit mille fois que vous étiez un Soleil; vous avez cru que vous deviez visiter toutes vos maisons l'une après l'autre, mais c'est ce qui vous trompe, Mademoiselle, car le Soleil ne bouge, et c'est la terre qui tourne (1663: 183-184). 
Comme l'illustre cet exemple, les galants épistoliers incorporent les idéaux précieux et courtois pour ensuite y donner un quolibet, ce trait d'esprit galant qui les relativise. Ainsi, ils contribuent à la redéfinition du sentiment amoureux, pour en même temps dépoussiérer son expression littéraire. Janet Paterson dénote en effet que l'incessante hybridation donne lieu à une charnière ou un noyau de nouveauté, où l'on oscille de "la désintégration d'une conception normative " du genre étudié à « l'avènement d'une autre forme d'écriture " (2001: 87-8). Nous constatons en effet que les entrecroisements féconds aux résonances ironiques typiquement galants créent de nouvelles formes et catégories.

Non seulement la lettre galante rajeunit le genre épistolaire en explorant les dimensions (auto-)fictionnelle et romanesque, mais encore elle renouvelle la littérature de lépoque en son intégralité par ses incessantes expériences avec le support textuel, l'intertextualité et la signature hybride. Fidèle à l'optique de Todorov qui présume que les genres viennent « tout simplement d'autres genres" (1987:30), nous supposons que la présence latente et inattendue du genre 'non-genre' des lettres galantes a été un catalyseur essentiel dans l'avènement du roman moderne. La pratique d'hybridation fait partie intégrante de la littérature même. Tout comme dans la nature, où l'hybridité désigne le croisement des espèces, l'hybridité crée dans les Lettres un produit non seulement novateur, mais également plus performant que ses éléments constitutifs.

\section{Conclusion}

À la découverte des lettres galantes produites au sein de l'Hôtel de Rambouillet, nous nous laissons surprendre par une forme épistolaire qui se situe entre le simple moyen de communication spontané et la composition littéraire savamment méditée. Les tensions présentes dans la forme de la lettre galante résonnent d'ailleurs dans l'identité de son scripteur qui défie un fragile équilibre entre le moi et le collectif, entre lécrivant et l'écrivain. Ces hybridités formelles et identitaires ne manquent pas de révéler les pas tantôt prudents, tantôt rebelles, d'un genre en voie de reconnaissance. Sa lutte nous instruit sur les enjeux majeurs de l'histoire littéraire du XVII ${ }^{e}$ siècle, telles les problématiques autour du statut de l'auteur et de la hiérarchie des genres, mais tout aussi bien sur les possibilités de création dans un champ littéraire en apparence rigide.

Lidée d'un public développait non seulement la conscience des modèles d'écriture, mais aussi l'envie de passer outre les modèles en faveur de l'originalité. La lettre galante, caractérisée par une hybridité générique éclatante, en devient le lieu de prédilection pour un métissage de genres, décomplexé et innovant. Les galants épistoliers ne se limitent point à faire coexister des genres. Ils déconstruisent leurs codes, mais pour les soumettre à une transformation tonifiante, revigorant par là leurs ressources poétiques. La lettre galante soffre à nous comme le spectacle poignant d'un combat 
de lécriture contre la convention, un lieu de lutte sans sueur ni poussière, où règnent l'alliage et l'inconstance, là où les codes se perdent dans la mêlée. Ses infimes effractions ont pollinisé des lignes littéraires trop droites, ouvrant ainsi la voie aux premiers romans « modernes » et, par extension, à la modernité. L'hybridité littéraire se prouve donc en effet révélatrice de cette modernité en ce quelle nous apprend sur les tensions à l’ouvre dans le champ littéraire, et innovatrice, en ce quelle ouvre en nouvelles perspectives par rapport à la création littéraire.

\section{BIBLIOGRAPHIE}

Allen M. 2013. Against 'Hybridity' in Genre Studies: Blending as an Alternative Approach to Generic Experimentation. Trespassing Journal [En ligne] 2. 3-21.

Balutet N. 2016. Du postmodernisme au post-humanisme : présent et futur du concept d'hybridité «Littératures et arts contemporains : l'hybridité à l'œuvre ». Babel. Littératures plurielles 33. URL : https://babel.revues.org/4084, consulté le 29/12/2016.

Batt N. et al. 2001. L'art et l'hybride. Saint-Denis. Presses universitaires de Vincennes.

Bernardot M. et Thomas H. 2014-2016. Notes sur l'hybridité. REVUE Asylon(s) 13. Transconcepts : lexique théorique du contemporain.

URL : http://www.reseau-terra.eu/article1327.html, consulté le 29/12/2016.

Boyer A. 1701 (éd.). Recueil de lettres françois \& anglois. Tirées des plus celébres autheurs françois... London. Daniel Brown.

Budor D. et W. Geerts (dir.). 2004. Le texte hybride. Paris. Presses Sorbonne Nouvelle.

Cotin C. 1663. Euvres galantes en prose et en vers de monsieur Cotin. Paris. E. Loyson.

Denis D. 1998. Ce que parler "prétieux " veut dire : Les enseignements d'une fiction linguistique au XVII' siècle. L'information grammaticale 78. 53-58.

Denis D. 2001. Le Parnasse Galant, institution d'une catégorie littéraire au XVII siècle. Paris. Honoré Champion.

Derrida J. 1986. Parages. Paris. Galilée.

Doumet Ch. et al. (dir.) 2001. L'Art et l'Hybride. Saint-Denis. Presses Universitaires de Vincennes. coll. «Esthétiques hors-cadre ».

Eigeldinger M. 1987. Mythologie et intertextualité. Genève. Slatkine.

Faret N. 1638 (éd.). Recueil de lettres nouvelles de messieurs Malherbe, Coulomby, Bois Robert, Molière, Plassac, Brun, Silhon, Godeau, Conac, Racan, Balzac, Auvray, et autres. Paris. A. Courbé.

Griem J. 1998. Hybridität. Metzler-Lexikon Literatur- und Kulturtheorie: Ansätze - Personen - Grundbegriffe. 3. 220-21.

Lathuillère R. 1962. Voiture et le «Bon usage » à l'Hôtel de Rambouillet. Cahiers de l'Association internationale des études françaises 14. 63-78.

Paterson J. 2001. Le paradoxe du postmodernisme : léclatement des genres et le ralliement du sens. In Dion R., Fortier F. \& Haghebaert E. Enjeux des genres dans les écritures contemporaines. Québec. Nota bene. 81-101.

Reynier C. 2004. Jeanette Winterson : Le miracle ordinaire. Pessac. Presses Universitaires de Bordeaux. 
Robert M. 1972. Roman des origines et origines du roman. Paris. Grasset.

Roux A. 1858 (éd.). Lettres du comte d'Avaux à Voiture, suivies de pièces inédites extraites des papiers de Conrart. Paris. A. Durand.

Schmitt A. 2015. Cognition et Hybridité. Le lecteur dans la zone grise. Cahiers de Narratologie 28. URL : https://narratologie.revues.org/7201, consulté le 30/12/2016.

Sinding M. 1995. Genera Mixta: Conceptual Blending and Mixed Genres in Ulysses. New Literary History 36 4. 589-619.

Todorov T. 1987. La notion de littérature et autres essais. Paris. Seuil.

Viala A. 1985. Naissance de l'écrivain. Sociologie de la littérature à l'âge classique. Paris. Minuit. Voiture V. 1691. Euvres. Lyon. Chize.

Voiture V. 1855. Euvres de Voiture : lettres et poésies. Ubicini J.-H.-A. (éd.). Paris. Charpentier. Young R. 1995. Colonial Desire: Hybridity in Theory, Culture and Race. London. Routledge.

\section{The gallant letter, or the fruitful abundance of transgression}

ABSTRACT: This essay concentrates on early modern expressions of literary hybridity in the $17^{\text {th }}$-century gallant letters of Vincent Voiture (1597-1648), Charles Cotin (1604-1681) and Antoine Godeau (1605-1672), circulating in the Parisian salon L'Hôtel de Rambouillet. Firstly, we look at the hybridity of the letter form an sich, within the context of salon sociability and early gallantry. Secondly, we study the multiplicity of both the authorial voice and the audience of the gallant letter. Thirdly, we highlight the intriguing exchange between genres within the gallant letter, as well as their confrontation and, finally, cross-fertilisation. This tripartite case study substantiates our conviction that literary hybridity functions as an intriguing indicator and catalyst of literary evolution and creation.

Keywords: Seventeenth-century letter writing, sociability, literary hybridity, gallant letter. 